\title{
Radiotéléphones cellulaires et santé : mise au point des recherches portant sur la santé chez l'homme
}

\author{
R. de SEZE*
}

(Manuscrit reçu le 18 mai 1999, accepté le 19 mai 1999)

\begin{abstract}
RÉSUMÉ Les radiotéléphones émettent un champ électromagnétique radiofréquence dont une partie est absorbée dans la tête de l'utilisateur. De nombreuses études ont été et sont encore réalisées pour étudier leur retentissement éventuel sur la santé humaine, aux niveaux expérimental, épidémiologique ou clinique. Après un bref rappel de dosimétrie, l'auteur fait le point de la recherche sur les effets graves et irréversibles, qui n'ont pas été confirmés, et sur quelques effets bénins qui semblent bien réels. La causalité du champ ékectromagnétique sur ces effets n'est cependant pas démontrée ; par ailleurs, des études sont en cours pour évaluer leurs éventuelles conséquences à long terme. Des interférences avec les stimulateurs cardiaques ont été observées, mais peuvent aisément être évitées en maintenant le téléphone portable à une distance supérieure à $10 \mathrm{~cm}$.
\end{abstract}

ABSTRACT Radiocellular phones and health: Up-to-date research on human health.

Abstract. Cellular telephones emit a radiofrequency electromagnetic field, part of which is absorbed in the user's head. Many studies have been conducted to look for their possible health hazard to human, at the experimental, epidemiological or clinical level. After a short recall on dosimetry, the author describes the state-of-the art about harmful and irreversible effects, up to now not confirmed, and about some benign symptoms which look absolutely actual. A causal relationship of the electromagnetic field on these benign symptoms is however not proven; other, studies are running to evaluate their possible long-term consequences. Interferences with cardiac pacemakers can easily be avoided by holding the telephone handset farer than $10 \mathrm{~cm}$.

\section{Introduction}

La radiotéléphonie cellulaire se développe depuis quelques années de façon considérable. Le public et les media se sont inquiétés des risques éventuels pour la santé de ce nouveau facteur environnemental. En effet, cette technique constitue une source importante d'exposition aux champs électromagnétiques radiofréquences, d'une part du fait de la proximité entre la source des champs et la tête, d'autre part du grand nombre d'utilisateurs actuels et potentiels. Parmi les préoccupations vis-à-vis de la

* Laboratoire de Biophysique Médicale - CHU de Nìmes - Faculté de Médecine. 30907 Nîmes Cedex 2, France. 
santé, deux niveaux peuvent être distingués :

- effets graves et irréversibles : en particulier, existe-t-il un effet déclenchant ou favorisant sur le cancer ? Jusqu'à présent, il n'a jamais été montré d'effet pathologique grave à court terme dû aux champs des radiotéléphones cellulaires. Si de tels effets existent, ils sont donc à long terme et devraient être cumulatifs ;

- effets bénins et réversibles : existe-t-il des effets moindres mais gênants pour la santé : sur le système nerveux (migraines, EEG, ..), sur le système endocrinien, sur le système immunitaire...

Pour répondre à la question des effets graves, deux types d'approche sont disponibles:

- l'expérimentation animale, qui a déjà été bien développée ; ses résultats ne sont pas toujours extrapolables à l'homme ;

- les études épidémiologiques, qui concernent directement l'homme, mais présentent également des inconvénients : facteurs confondants, faible nombre de cas, longue période de latence avant obtention des résultats.

Pour évaluer les effets bénins, le meilleur modèle, permettant d'éluder les risques d'extrapolation inappropriée, reste l'expérimentation humaine, pour laquelle nous différencierons également 2 types :

$\Rightarrow$ détection d'un effet physiologique, permettant de mettre en évidence la sensibilité de l'organisme au champ électromagnétique émis ;

$\Rightarrow$ recherche d'un effet néfaste pour la santé : mise en évidence de la causalité entre les symptômes décrits, des céphalées par exemple, et l'exposition aux radiotéléphones.

\section{Avancement de la recherche}

L'évolution des connaissances ces dernières années est la suivante :

\subsection{Considérations physiques}

\section{Paramètres d'émission des téléphones}

Les paramètres des champs émis par les téléphones mobiles sont standardisés selon différents systèmes en fonction des régions et des pays. En France, les 2 systèmes 
actuellement en place sont le système GSM 900, commercialisé par les opérateurs Itineris et SFR, et le système GSM 1800 , plus récent, commercialisé par l'opérateur Bouygues Telecom.

\section{Système GSM 900 (Global System for Mobile)}

La fréquence porteuse est dans la gamme des $900 \mathrm{MHz}$. En fait, elle s'étend de 890 à $915 \mathrm{MHz}$; à l'intérieur de cette gamme, les bases cellulaires attribuent à chaque utilisateur une bande plus étroite de $0,2 \mathrm{MHz}$ pour chaque communication (découpage fréquentiel). Cette bande est aléatoire et peut notamment être amenée à changer lorsque l'utilisateur se déplace ; sa communication est alors relayée d'une base à une autre.

À l'intérieur de chaque bande de fréquence, il existe un nouveau découpage, temporel cette fois-ci : l'information est émise par impulsions, à raison d'une impulsion de $576 \mu$ s toutes les $4,6 \mathrm{~ms}$ (fréquence de répétition des impulsions : $217 \mathrm{~Hz}$; rapport cyclique de 1/8). Ceci permet que chaque bande de fréquence soit utilisée à tour de rôle par 8 utilisateurs différents. La communication est ensuite « reconstituée », après décodage, le tout dans un temps suffisamment court pour qu'elle semble continue. Une conséquence intéressante de ce fractionnement temporel vis-à-vis de l'interaction avec l'utilisateur est une diminution proportionnelle de la puissance moyenne $P_{\text {moy }}$ par rapport à la puissance-crête $P_{c}$, qui est de $2 \mathrm{~W}$ :

$$
P_{\text {moy }}=P_{\mathrm{c}} / 8=2 \mathrm{~W} / 8=0,25 \mathrm{~W} \text { ou } 250 \mathrm{~mW} \text {. }
$$

\section{Champ émis, SAR}

Il n'est pas suffisant de connaître les paramètres d'émission tels que la puissance émise ou le champ électrique, pour évaluer la puissance absorbée par l'utilisateur. Environ $40 \%$ seulement de l'énergie émise est absorbée dans la tête. Une unité de dose absorbée de champ électromagnétique est le débit d'absorption spécifique (DAS, plus connu sous le terme anglo-saxon équivalent $S A R$ ). Le $S A R$ correspond à la puissance absorbée en watts (W), divisée par la masse de tissu en $\mathrm{kg}$ dans laquelle cette puissance se répartit. Il s'exprime donc en W/kg. Une première approximation de la puissance absorbée moyennée sur l'ensemble de la tête $S A R_{\text {moy }}$ est donc, si l'on considère une tête de masse $M=3 \mathrm{~kg}$, de :

$$
S A R_{\text {moy }}=P_{\text {moy }} \times 40 \% / M=250 \times 40 \% / 3=30 \mathrm{~mW} / \mathrm{kg}, \text { ou } 0,03 \mathrm{~W} / \mathrm{kg} .
$$

Cependant, l'absorption dans la tête n'est pas homogène : $70 \%$ de l'énergie absorbée l'est dans un volume de $5 \mathrm{~cm}$ de côté, soit dans une masse de tissu d'environ $125 \mathrm{~g}$. Il en résulte que le $S A R$ local $S A R_{\text {loc }}$ est supérieur, à savoir :

$$
S A R_{\mathrm{loc}}=0,25 \times 40 \% \times 70 \% / 0,125=0,6 \mathrm{~W} / \mathrm{kg} \text {. }
$$


La puissance absorbée décroît exponentiellement en fonction de la profondeur par rapport à celle existant en surface. Le SAR sous-cutané peut donc être encore supérieur lorsqu'il est calculé sur un petit volume ( $1 \mathrm{~g} \mathrm{ou} 10 \mathrm{~g})$. On est alors obligé pour calculer ce $S A R$ de connaître ou de calculer la valeur du champ électrique $E_{\text {int }}$ dans les tissus, selon la formule :

$S A R=\sigma E_{\text {int }}{ }^{2} / \rho$, où $\sigma$ est la conductivité des tissus biologiques et $\rho$ leur masse volumique.

À $900 \mathrm{MHz}$, une valeur moyenne de $\sigma$ pour les tissus biologiques est de l'ordre de 1 siemens ou (ohm.m) $)^{-1}$ (S ou $\left.(\Omega . m)^{-1}\right) ; \rho$ est de l'ordre de $1000 \mathrm{~kg} / \mathrm{m}^{3}$. Il n'y a pas de relation simple entre le champ émis par le téléphone en l'absence de la tête et le champ dans les tissus. Celui-ci doit être calculé par modélisation. Anderson et Joyner (1995) ont trouvé une valeur maximum du champ interne autour de $30 \mathrm{~V} / \mathrm{m}$ pour une puissance d'émission de $0,6 \mathrm{~W}$, c'est-à-dire $50 \mathrm{~V} / \mathrm{m}$ pour $1 \mathrm{~W}$ émis. Ce champ correspond à un $S A R$ de $2,5 \mathrm{~W} / \mathrm{kg}$ pour $1 \mathrm{~W}$ émis, ce qui est bien corrélé à la valeur précédemment trouvée de $0,6 \mathrm{~W} / \mathrm{kg}$ pour $250 \mathrm{~mW}$. Différentes autres simulations aboutissent à des valeurs comprises entre 0,4 et un peu plus de $1 \mathrm{~W} / \mathrm{kg}$.

Deux contraintes contribuent à minimiser le $S A R$ dans la tête des utilisateurs :

- l'efficacité optimale des radiotéléphones implique que la majorité du rayonnement soit émis en direction de la station de base et donc que la partie absorbée par la tête de l'utilisateur soit minimale ;

- l'autonomie maximale et le faible poids de la batterie seront assurés par l'utilisation de la plus faible quantité d'énergie possible pour les communications, donc de la puissance d'émission minimale.

\section{Paramètres d'émission des stations de base}

Les stations de base se multiplient et suscitent des réactions de rejet de la part des individus habitant à proximité. On distingue les stations micro-cellulaires, moins puissantes, et les stations macro-cellulaires. Du point de vue physique, le champ auquel peuvent être soumis des personnes se présente de la façon suivante :

- Strictement en face de l'antenne, le champ à une distance de 1 mètre d'une station micro-cellulaire est de $50 \mathrm{~V} / \mathrm{m}$, puis décroît exponentiellement. Ceci nécessite de se tenir à une distance minimale de $1,5 \mathrm{~m}$ pour respecter la recommandation européenne ENV 50166-2 qui est de $41 \mathrm{~V} / \mathrm{m}$ à $900 \mathrm{MHz}$ et de $58 \mathrm{~V} / \mathrm{m}$ à $1800 \mathrm{MHz}$. Cette distance est de $2,5 \mathrm{~m}$ pour une station macro-cellulaire.

- En arrière de l'antenne, une plaque métallique absorbe la majorité des champs qui pourraient y être émis. Une distance de $50 \mathrm{~cm}$ est cependant recommandée pour garantir le respect de ces normes. 
- Dès que l'on s'éloigne de l'axe de l'antenne au-dessus ou en dessous (cas le plus courant, ces antennes êtant habituellement disposées à une hauteur de 20 m environ), le champ est au maximum de 1 à $2 \mathrm{~V} / \mathrm{m}$, ce maximum étant situé à une distance horizontale de 10 à $20 \mathrm{~m}$ du pylône supportant l'antenne.

Il ressort de ces éléments que dans la majorité des cas, l'exposition humaine par les stations de base est négligeable et ne constitue donc pas une préoccupation scientifique et de santé prioritaire.

\subsection{Considérations biologiques : santé, médecine}

\section{Expérimentations animales signifiantes}

La majorité des expérimentations animales réalisées jusqu'à présent n'apportent pas d'argument étayant les craintes émises vis-à-vis du cancer. En particulier, il n'a pas été montré que les champs émis par les radiotéléphones cellulaires puissent initier (déclencher) un cancer. Des travaux portant sur de multiples modèles confortent cette tendance :

- un test classique, d'exposition chronique sur deux ans, consiste à vérifier au décours d'une exposition durant toute la vie de l'animal, la fréquence de tumeurs apparues et responsables du décès de l'animal en la comparant à la fréquence de tumeurs spontanées dans une population témoin ; les études de Adey et al. (1999) et Zook (1998) montrent des résultats négatifs (pas d'effet de l'exposition) et d'autres sont en cours (Roti-Roti, Anderson).

Des modèles de cancer ont été développés pour augmenter la sensibilité éventuelle à un agent extérieur, soit après induction chimique à différents facteurs (Ethyl Nitroso-Urée ou ENU, Benzo-alpha Pyrène ou $B \alpha P$, Di-Méthyl BenzAnthracène ou DMBA, Di-EthylNitrosamine ou DEN), parfois associés à un élément co-promoteur (PhenoBarbital, 12-o-TetradecanoylPhorbol-13-Acetate TPA), soit après irradiation ou après modification génétique. Plusieurs études n’ont pas montré d'augmentation du pourcentage de tumeurs chez les animaux exposés (Adey et al., 1999), sur le cancer du cerveau après injection pendant la gestation d'Ethyl Nitroso-Urée ; cette étude est en cours de réplication par Zook et al.; Chagnaud et al., 1995 sur un sarcome induit par le benzo alpha pyrène ; Imaida et al., 1998a, b sur une tumeur hépatique induite par la Diéthylnitrosamine ; Heikkinen et al., 1998, sur des tumeurs induites par une irradiation de 4 Gy). D'autres sont en cours avec du DMBA. Seuls les travaux de Szmigielski et Szudzinski (1982) ont montré une accélération du développement tumoral, mais avec des doses supérieures à $6 \mathrm{~W} / \mathrm{kg}$ pendant plusieurs mois.

- La greffe de tumeurs syngéniques permet d'étudier l'effet des champs radiofréquences sur la prolifération. Aucun effet n'a été observé avec les cellules de gliome 9L (Higashikubo et al., 1998) ou RG-2 (Salford et al., 1993). 
Cependant, deux résultats, non confirmés, ne permettent pas d'éliminer complètement l'hypothèse d'un risque pour la santé :

- les travaux de Lai $(1995,1996)$ montrent une fragilité accrue des chromosomes de cellules nerveuses de rats après une exposition $(2,45 \mathrm{GHz}$, impulsions de $2 \mu$ s toutes les $2 \mathrm{~ms}$ (500 pps)) (inhibition des systèmes physiologiques de réparation). Cette étude n'a pas été confirmée par d'autres équipes (Tice et al., Malyapa et al. 1997a et b; 1998), mais elle est en cours de réplication par les auteurs eux-mêmes ;

- l'étude de Repacholi (1997) a montré une incidence augmentée (x 2,4) de lymphomes chez des souris génétiquement prédisposées (souris transgéniques E $\mu$-pim1) après exposition chronique au champ des radiotéléphones. Cette expérience est en cours de réplication (Kuchel, Australie). En cas de confirmation, il faudrait en rechercher les mécanismes ou/et les conséquences éventuelles sur la santé humaine.

\section{Études chez l'homme}

Une hypothèse qui gouverne la majorité des études sur volontaires sains est la suivante : si on craint un effet grave à long terme, il doit exister une interaction primaire avec l'organisme à l'origine de changements dont les répercussions à long terme « pourraient » être graves. Ce sont ces interactions primaires que l'on cherche à mettre en évidence dans ces études. La revue de la littérature sur les effets biologiques d'une exposition aux rayonnements radiofréquences indique que trois systèmes sont particulièrement susceptibles de révéler une interaction : le système nerveux ; le système endocrinien ; le système immunitaire.

Peu d'études ont exploré jusqu'à présent le système immunitaire, mais plusieurs études chez l'homme ont porté sur le système endocrinien et le système nerveux.

\section{Système endocrinien}

Après exposition à des radiotéléphones cellulaires $2 \mathrm{~h} /$ jour pendant 4 semaines, il n'a pas été observé de différence significative sur les sécrétions de base ou les rythmes circadiens des hormones suivantes : ACTH, cortisol, LH, FSH, GH (hormone de croissance), TSH (hormone de stimulation de la thyroïde) et prolactine (de Seze et al., 1998 $\mathrm{a}, \mathrm{b})$. Une autre hormone intéressante est la mélatonine, pour laquelle une inhibition de la sécrétion sous l'effet des champs électromagnétiques a été montrée par plusieurs équipes chez l'animal. Un tel effet n'a pas été retrouvé chez l'homme après exposition aux champs des téléphones mobiles (de Seze et al., 1997 ; 1999 ; Burch, 1997 ; Vollrath, études en cours). Plusieurs études n'ont pas trouvé d'effet chez l'animal (Vollrath et al., 1997 ; Lerchl et al., 1998 ; Kumlin et al., 1997 ; Buschmann, en cours). 


\section{Système nerveux}

\section{Système nerveux : PEA et PDOE}

L'organe le plus exposé aux champs des radiotéléphones est l'oreille. Les études réalisées à l'aide de techniques d'enregistrement les plus sensibles possible ont recherché une perturbation de signaux électrophysiologiques stimulés de cet organe par une exposition d'une heure aux champs des radiotéléphones : ce sont les potentiels évoqués auditifs (PEA) et les produits de distorsion des oto-émissions (PDOE). Une modification a été observée par Kim et al. (1998) sur les PEA, mais pas par Thimonier et al. (1997 a, b ); et de Seze et al. (1998 c).

\section{Système nerveux : électroencéphalogramme (EEG)}

Un autre signal susceptible d'être perturbé par une stimulation extérieure est l'électroencéphalogramme (EEG). Une modification de ce signal a été recherchée après une heure d'exposition. Afin de sensibiliser au mieux cet examen, l'analyse a été réalisée après numérisation de l'EEG (EEG quantifié), permettant de rechercher un décalage discret des fréquences ou une modification de l'amplitude d'un signal à une fréquence déterminée. Le paramètre quantifié dans ce cas est la puissance spectrale par bande de fréquence.

Il a été trouvé une légère modification de l'activité électrique cérébrale, dont l'interprétation physiologique est en cours afin d'évaluer l'impact de cette modification sur la santé, qui ne semble pas a priori pouvoir être nocif.

D'autres équipes ont également étudié l'influence des radiotéléphones sur l'EEG (Eulitz et al., 1998 ; Freude et al., 1998 ; Dec et al., 1997). Thuroczy a trouvé un effet d'abord chez le rat (1995), puis chez l'homme (Bologne, 1997). Röschke et Mann ont également publié un effet chez l'homme pendant le sommeil, l'exposition durant toute la nuit. La latence d'endormissement était légèrement réduite (9 min au lieu de $12 \mathrm{~min}$ ) et la durée totale du sommeil paradoxal (REM) était également diminuée (Mann et Röschke, 1996). Au cours d'une deuxième étude avec un système d'exposition « mieux contrôlé », légèrement différent bien qu'un peu plus puissant, cet effet n'a pas été confirmé (Wagner et al., 1998). Par contre, aucun effet n'a été observé chez des volontaires éveillés soumis à une exposition de quelques minutes (Röschke et Mann, 1997 ; Hietanen et al., 1997 ; Spittler et al., 1997). De nombreuses autres études sont en cours, avec une sensibilisation par des tests cognitifs pour évaluer en plus d'un effet électrophysiologique, le retentissement fonctionnel éventuel des expositions réalisées.

Un autre paramètre régulateur important du système nerveux, la perméabilité de la barrière hémato-encéphalique, a également fait l'objet de plusieurs études, mais nécessite actuellement des confurmations. 


\section{Épidémiologie}

Une étude épidémiologique a été initiée par Rothman, comparant la mortalité entre des utilisateurs de radiotéléphone mobile et des utilisateurs de radiotéléphone portable (Rothman, 1996a). Un portable a son antenne proche de la tête et expose donc son utilisateur au champ de radiocommunication, tandis que l'antenne d'un mobile est déportée, en général sur le toit ou le capot du véhicule, et son utilisateur n'est pas directement exposé à un champ radiofréquence significatif. À court terme sur la seule année 1994, les résultats ne montrent pas de différence significative entre ces deux groupes, mais il faudra vraisemblablement attendre plusieurs années pour disposer de résultats interprétables. De multiples précautions ont été prises pour définir et prendre en compte les facteurs confondants possibles (Rothman, 1996b), et préciser les paramètres utilisables pour évaluer au mieux l'exposition (Bren, 1996). Il faut cependant noter que cette étude est réalisée aux États-Unis où $80 \%$ des systèmes cellulaires fonctionnent avec des signaux analogiques, pour lesquels le codage est très différent des signaux numériques principalement utilisés en Europe (analogique : codage principalement par modulation de fréquence et d'amplitude sur une émission continue ; numérique : codage par modulation de fréquence sur une émission par impulsions).

En ce qui concerne l'article de Davidson paru le 5 janvier 1998 dans le Medical Journal of Australia, il faut souligner qu'il ne s'agit pas d'une étude épidémiologique. C'est une lettre à l'éditeur qui fait état d'une observation : l'incidence des tumeurs du cerveau a augmenté de 1982 à 1992, période pendant laquelle les radiotéléphones cellulaires se sont répandus. Davidson émet l'hypothèse d'une possible corrélation qu'il conviendrait d'étudier. S'il est exact qu'il ne faut pas négliger cette possibilité, de nombreux autres facteurs peuvent être à l'origine de cette augmentation, ne seraitce que le SIDA, qui s'est développé pendant la même période et qui peut générer des tumeurs du cerveau.

Dans une étude plus récente, Mild recherchait s'il existait une différence dans les symptômes ressentis entre les utilisateurs de radiotéléphones analogiques et ceux utilisant des radiotéléphones numériques. Sans que les différences entre ces deux groupes soient importantes, il ressort de cette étude que certains symptômes tels que des crises de migraine, de l'asthénie et une sensation d'échauffement cutané sont plus fréquents en fonction de la durée d'utilisation, par rapport à un groupe de référence exposé moins de 2 min. par jour (Oftedal et al., 1998a et b).

Une autre étude à grande échelle est en préparation dans le cadre du $5^{\mathrm{e}}$ programme de recherche et développement technologique de l'Union européenne par le Centre international de recherche contre le cancer (IARC, Lyon). Elle portera principalement sur les tumeurs du cerveau, de la face et du cou, mais prendra également en compte d'éventuels symptômes que pourraient ressentir les utilisateurs. 


\section{Stimulateurs cardiaques}

Le risque le plus objectif sur la santé humaine est cependant celui d'interférences avec les pace-makers chez les sujets appareillés. Bien que le risque en situation normale d'utilisation soit minime et ait été principalement observé dans des conditions où le pace-maker n'était pas implanté (essais in vitro), il est nécessaire de prévenir les porteurs de pace-makers de ce risque afin qu'ils n'approchent pas leur téléphone à moins de $15 \mathrm{~cm}$ de leur poitrine, même et surtout en veille, et qu'ils l'utilisent sur l'oreille contro-latérale au côté d'implantation du pace-maker. Aucune interférence n'a été observée lorsque les radiotéléphones étaient tenus à plus de $10 \mathrm{~cm}$ des stimulateurs. Le champ à $900 \mathrm{MHz}$ émis par un radiotéléphone GSM de $2 \mathrm{~W}$ crête est à cette distance de l'ordre de $80 \mathrm{~V} / \mathrm{m}$. Ces interférences peuvent éventuellement être testées dans un service de cardiologie.

\section{Conclusions et perspectives}

L'état actuel des connaissances quant au risque de cancer chez l'homme de par l'utilisation de radiotéléphones cellulaires est rassurant (Juutilainen et de Seze, 1998). Un risque pour la santé ne peut pas être totalement exclu, mais il semble, si les expérimentions animales qui le font craindre sont confirmées, qu'il ne se manifeste que dans des circonstances bien particulières qu'il resterait alors à définir. D'autres manifestations pathologiques de gravité moindre telles que des céphalées pourraient être dues à l'utilisation de cette technologie. De légères modifications de l'électrogenèse cérébrale nécessitent également d'être confirmées ; si c'est le cas, les conséquences possibles de ces perturbations sur la santé humaine devront être recherchées (Repacholi et al., 1998).

Remerciements : Ce travail a été réalisé dans le cadre d'une convention avec l'INRS.

\section{RÉFÉRENCES}

Adey W.R., Byus C.V., Cain C.D., Higgins R.J., Jones R.A., Kean C.J., Kuster N., MacMurray A., Stagg R.B., Zimmerman G., Phillips J.L. and Haggren W. (1999) Spontaneous and nitrosourea-induced primary tumors of the central nervous system in Fisher 344 rats chronically exposed to $836 \mathrm{MHz}$ modulated microwaves. Radiat. Res. 152, 293-302.

Anderson V, Joyner K.H. (1995) Specific absorption rate levels measured in a phantom head exposed to radiofrequency transmissions from analog hand-held mobile phones Bioelectromagn. 16, 60-69.

Bren S.P.A. (1996) Reviewing the RF safety issue in cellular telephones. IEEE Eng. Med. Biol. Mag. 15, 109-115.

Burch J.B., Reif J.S., Pitrat C.A., Keefe T.J., Yost M.G. (1997) Cellular telephone use and excretion of a urinary melatonin metabolite. Presented at 1997 Ann. Rev. Res. Biol. Effects of Electric \& Mag. Fields, San Diego, CA. 
Chagnaud J-L., Veyret B., Despres B. (1995) Effects of pulsed microwaves on chemically-induced tumors in rats (mecting abstract). Bioelectromagnetics Society, 17th Annual Meeting, 18-22 June, Boston, $M A$, p. 28 .

Dec S., Cieslak E., Miszczak J.S. (1997) Electroencefalographic frequency mapping in healthy subjects during cellular head telephone stimulation. 2nd World Congress for Electricity and Magnetism in Biology and Medicine, Bologna, Italy.

de Seze R., Ayoub J., Fabbro-Peray P., Miro L., Touitou Y. (1997a) Étude de l'exposition aux champs électromagnétiques émis par les téléphones mobiles GSM et DCS sur le rythme circadien de mélatonine chez l'homme. Congrès National de Radioprotection, SFRP 1997 Avignon, 18-22/06.

de Seze R., Ayoub J., Chabert R., Thimonier C., Lallemant J.-G., Miro L. (1997b) Étude chez l'homme de l'exposition aux champs électromagnétiques émis par les téléphones mobiles GSM sur les potentiels évoqués auditifs et les produits de distorsion acoustiques. Congrès National de Radioprotection, SFRP 1997, Avignon pp. 47-51.

de Seze R., Fabbro-Peray P., Miro L. (1998a) GSM radiocellular telephones do not disturb the secretion of antepituitary hormones in human. Bioelectromagn. 19, 271-278.

de Seze R., Ayoub J., Miro L. Touitou Y. (1998b) Effect of electromagnetic fields emitred by mobile telephones on endocrine circadian rhythms in humans. In Biological Clocks: Mechanisms and Applications. Excerpta Medica Int Cong Series 1152. Yvan Touitou Ed. Elsevier, Oxford, pp. 313-316.

de Scze R., Ayoub J., Fabbro-Peray P., Miro L. Touitou Y. (1999) Evaluation in humans of the effects of radiocellular telephones on the circadian patterns of melatonin secretion, a chronobiological rhythm marker (accepted).

Eulitz C., Ullsperger P., Freude G., Elbert T. (1998) Mobile phones modulate response patterns of human brain activity. Neuroreport $9,3229-3232$.

Freude G., Ullsperger P., Eggert S., Ruppe (1998) Effects of microwaves emitted by cellular phones on human slow brain potentials. Bioelectromagn. 19, 384-387.

Heikkinen P., Kosma V-M., Hongisto T., Huuskonen H., Hyysalo P., Komulainen H., Kumlin T., Lahtinen T., Lang S., Penttila I., Juutilainen J. (1998) Effects of radiofrequency radiation on the development of cancer in mice. Bioelectromagn. Society Meeting, June 7-11, 1998, St. Petersburg, FL.

Hietanen M., Kovala T., Hamalainen AM., Velin R., von Nandelstadh P. (1997) EEG activity of the human brain during exposure to ccllular phones. 2 nd World Congress for Electricity and Magnetism in Biology and Medicine, Bologna, Italy.

Higashikubo H., Culbreth V.O., Spitz D.R., Pickard W.F., LaRegina M., Straube W.L., Moros E., Gutting K.M., Kuepfert H.J., Roti Roti J.L. (1999) Effect of radio frequency electromagnetic field on the in vivo proliferation of 9L brain tumor. Radiat. Res. (submitted).

Imaida K., Taki M., Yamaguchi T., Ito T., Watanabe S., Wake K., Aimoto A., Kamimura Y., Shirai T. (1998a) Lack of promoting effects of the electromagnetic near-field used for cellular phones (929 MHz) on rat liver carcinogenesis in medium-term bioassay. Carcinogenesis 19, 311-314.

Imaida K., Taki M., Watanabe S., Wake K., Kamimura Y., Shirai T. (1998b) The electromagnetic near field used for cellular phones $(1.5 \mathrm{GHz})$ does not promote liver carcinogenesis in a medium term liver bioassay using F344 male rats. Bioelectromagn. Society Meeting, June 7-11, 1998, St. Petersburg, $F L$

Juutilainen J., de Scze R. ( 1998) Biological effects of amplitude-modulated radiofrequency radiation. Scand J. of Work, Environment and Health, 24, 245-54.

Kim Y.S., Cho Y.S., Kim S.I., Kim M.H. (1998) Characteristics of EEG and AEP in human voluntecrs exposed to RF. Bioelectromagn. Society 20th Annual Meeting, St. Petersburg, FL. 


\section{RADIOTÉLÉPHONES CELLULAIRES ET SANTÉ}

Kumlin T., Heikkinen P., Laitinen J., Juutilainen J. ( 1997) Nocturnal secretion of 6-hydroxy-melatonin sulphate in mice exposed to $900 \mathrm{MHz}$ radiofrequency radiation or $50 \mathrm{~Hz}$ magnetic fields. Second World Congress for Electricity and Magnetism in Biology and Medicine, 8-13 June, Bologna, Italy, p. 147.

Lai H. Singh N.P. (1995) Acute low intensity microwave exposure increases DNA single-strand breaks in rat brain cells. Bioelectromagn. 16, 207-210.

Lai H., Singh N.P. (1996) Single- and double-strand DNA breaks in rat brain cells after acute exposure to radiofrequency electromagnetic radiation. Int. J. Radiat. Biol. 69, 513-521.

Lerchl A., Brendel H., Streckert JF., Bitz A.K., Hansen V.W. (1998) Investigations on the Effects of 900 MHz Electromagnetic Fields on Growth, Melatonin, and Testicular Cell Composition in Djungarian Hamsters. Bioelectromagn. Society 20th Annual Meeting, St. Petersburg, FL.

Malyapa R.S., Ahern E.W., Straube W.L., Moros E.G., Pickard W.F., Roti-Roti J.L. (1997a) Measurement of DNA damage after exposure to $2450 \mathrm{MHz}$ electromagnetic radiation. Radiat. Res. 148, 608617.

Malyapa R.S., Ahern E.W., Straube W.L., Moros E.G., Pickard W.E., Roti-Roti J.L. (1997b) Measurement of DNA damage after exposure to clectromagnetic radiation in the cellular phone communication band. Radiat. Res. $148,618-627$.

Malyapa R.S., Ahem E.W., Bi C., Straube W.L., LaRegina M., Pickard W.E., Roti-Roti J.L. (1998) Measurement of DNA damage in rat brain cells after in vivo exposure to $2450 \mathrm{MHz}$ electromagnetic radiation and various methods of euthanasia. Radiat. Res. 149, 637-645.

Mann K., Röschke J. (1996) Effects of pulsed high-frequency electromagnetic fields on human sleep. Neuropsychobiol. 33, 41-47.

Oftedal G., Sandstrom M., Hansson Mild K., Wilen J. (1998a) Symptoms experienced in connection with use of mobile phones. A Swedish-Norwegian epidemiological study (meeting abstract). Bioelectromagn. Society, 20th Annual Meeting, 7-1/ June, St. Pete Beach, FL, Abstract No. B-11, p. 51.

Oftedal G., Sandstrom M., Wilen J., Mild K. H. (1998b) Subjective symptoms among mobile phone users in Sweden and Norway and measures taken to reduce the symptoms (Mceting Abstract). European Bioelectromagn. Assoc: (EBEA), 4th International Congress, 19-21 November, Zagreb, Croatia.

Repacholi M., R. de Sezc et al (1998) Low-level cxposure to radiofrequency ficlds : health effects and research needs. Bioelectromagn. 19, 1-19

Repacholi M.H., Basten A., Gcbski V., Noonan D., Finnic J., Harris A.W. (1997) Lymphomas in E $\mu$-Pim 1 transgenic mice exposed to pulsed $900 \mathrm{MHz}$ electromagnetic fields. Radiat. Res. 147, 631-640.

Röschke J. Mann K. (1997) No short-term effects of digital mobile radio telephone on the awake human electroencephalogram. Bioelectromagn. 18, 172-176.

Rothman K.J., Chou C.-K., Morgan R., Balzano Q., Guy A.W., Funch D.P., Preston-Martin S., Mandel J., Steffens R., Carlo G. (1996a) Assessment of cellular telephone and other radio frequency exposure for epidemiologic research. Epidemiology 7, 291-298.

Rothman K.J., Loughlin J.E., Funch D.P., Dreyer N.A. (1996b) Overall mortality of cellular telephone customers, Epidemiology 7, 303-305.

Salford L.G., Brun A., Persson B.R.R., Eberhardt J. (1993) Experimental studies of brain tumor development during exposure to continuous and pulsed $915 \mathrm{MHz}$ radiofrequency radiation. Bioelectrochem. and Bioenerg. 30, 313-318.

Spittler J.F., Calabrese P., Gehlen W. (1997) Cerebro-biological effects in low frequency pulsed RF fields. 2nd World Congress for Electricity and Magnetism in Biology and Medicine, Bologna, Italy. 
Szmigielski S., Szudzinski A., Pietraszek A., Bielec M., Janiak M., Wrembel J.K. (1982) Accelerated development of spontaneous and benzopyrene-induced skin cancer in mice exposed to $2450-\mathrm{MHz}$ microwave radiation. Bioelectromagn. 3, 179-191.

Szudzinski A., Pietraszek A., Janiak M., Wrembel J., Kalczak M., Szmigielski S. (1982) Acceleration of the development of benzopyrene-induced skin cancer in mice by microwave radiation. Arch. Dermatol. Res. 274, 303-312.

Thimonier C., Chabert R., Ayoub J., de Seze R., Lallemant J.-G., Miro L. (1997a) Étude chez l'homme de l'exposition aux champs électromagnétiques émis par les téléphones mobiles GSM sur les potentiels évoqués auditifs et les produits de distorsion acoustiques. Congrès National de RadioProtection, Avignon, 18-22 juin 1997.

Thimonier C., Chabert R., Ayoub J., de Seze R., Lallemant J.-G., Miro L. (1997a) No effect in humans of microwaves emitted by GSM mobile telephones on the auditory brainstem responses and auditory distortion products. Second World Congress for Electricity and Magnetism in Biology and Medicine, Bologna, June 1997, pp. 273-274.

Thuroczy G., Kubinyi G., Nagy N., Szabo L.D. (1995) Measurements of visual evoked potentiels (VEP) and brain electrical activity (EEG) after GSM-type modulated microwave exposure on rats. Honma T., Ed. In: Advanced Computational Electromagnetics. Amsterdam: Elsevier/IOS Press pp. 384 395.

Thuroczy G., Kubinyi G., Sinay H., Bakos J., Sipos K., Lenart A., Szabo L.D. (1997) Human studies on potential influence of RF exposure emitted by GSM cellular phones on cerebral circulation and electroencephalogram (EEG). Second world congress for electricity and magnetism in biology and medicine June 8-13, pp. 164-165.

Vollrath L., Spessert R., Kratzsch T., Keiner M., Hollmann H. (1997) No short-term effects of high-frequency electromagnetic fields on the mammalian pineal gland. Bioelectromagnetics 18, 376-387.

Wagner P., Röschke J., Mann K., Hiller W., Frank C. (1998) Human sleep under the influence of pulsed radiofrequency electromagnetic fields: a polysomnographic study using standardized conditions. Bioelectromagn. 19, 199-202.

Zook B. (1998) Radiofrequency irradiation of the brain of rats. Bioelectromagn. Society 20th Annual Meeting, St. Petersburg, FL. 